\title{
ULTERIORE STUDIO SULLE OSCILLAZIONI LIBERE DEL LAGO DI SCANNO
}

\author{
P. Caloi - D. Di Filippo - M. C. Spadea
}

D. Di Filippo ha dedicato un lavoro (') allo studio delle oscillazioni libere del lago di Scanno, al quale ha applicato i metodi di Hidaka e di Defant.

Dalla forma della curva normale, riportata nella fig. 12, parve possibile pervenire alle caratteristiche idrodinamiche del lago in questione, applicando uno dei metodi suggeriti da Chrystal, e precisamente quello che considera la curva normale formata da tratti rettilinei raccordati.

Si sa che, in un lago a fondo rettilineo, dove la profondità in un punto $x$ è data da $h\left(1-\frac{x}{a}\right)$, gli spostamenti orizzontali $\xi$ e verticali $\zeta$ di una particella alla superficie libera sono espressi da $\left(^{2}\right)$

$$
\begin{aligned}
\xi(\omega) & =\left\{A J_{\mathrm{q}}(\omega)+B Y_{1}(\omega) ! \sin n(t-\tau)\right. \\
\zeta & =\frac{\underline{s}}{2 a}\left\{A J_{\mathrm{o}}(\omega)+B Y_{\mathrm{o}}(\omega)\right\} \sin n(t-\tau)
\end{aligned}
$$

dove

$$
n=\frac{2 \pi}{T} \quad, \quad \omega=\frac{2 n a}{\sqrt{g h}} \sqrt{1-\frac{x}{a}}
$$

A e B essendo costanti arbitrarie e $J_{\mathrm{m}}(\omega), Y_{\mathrm{m}}(\omega)$ funzioni di Bessel e di Neumann di ordine $m$.

Vediamo di estendere ora questa teoria al caso rappresentato dalfig. 1 .

Sia $h$ la minima profondità nel punto $C$ e $h_{1}, h_{2}$, le massime profondità in $\mathrm{B}$ e in $\mathrm{D}$. Sia $\mathrm{AB}=p_{1}^{\prime}, \mathrm{BC}=p_{1}, \mathrm{CD}=p_{2}, \mathrm{DE}=p^{\prime}{ }_{2}$. Sia inoltre $h_{1}\left(1-\frac{x}{a_{1}{ }^{\prime}}\right)$ la legge di profondità per $\mathrm{AB}$ e $h_{1}\left(1+\frac{x}{a_{2}{ }^{\prime}}\right)$ quella per $\mathrm{BC}$, dove $x$ è misurato entrambe le volte da $\mathrm{B}$, positivamente da $\mathrm{B}$ verso $\mathrm{A} ; h_{2}\left(1-\frac{x}{a_{0}}\right)$ per $\mathrm{CD}, h_{2}\left(1+\frac{x}{a_{a}{ }^{\prime}}\right)$ per $\mathrm{DE}$, dove $x$ è misurato entrambe le volte da $D$, positivamente da $D$ verso C. Allora le profondità in $\mathrm{A}, \mathrm{C}, \mathrm{E}$ richiedono che sia 


$$
\begin{aligned}
& p_{1}^{\prime}=a_{1}^{\prime} \quad ; \quad h=h_{1}\left(1-\frac{p_{1}}{a_{1}}\right)=h_{2}\left(1-\frac{p_{2}}{a_{2}}\right) \quad ; \quad p_{2}{ }^{\prime}=a_{2}{ }^{\prime} \quad ; \\
& p_{1}+p_{2}=a_{1}\left(1-\frac{h}{h_{1}}\right)+a_{2}\left(1-\frac{h}{h_{\mathrm{a}}}\right)
\end{aligned}
$$

ciò che determina compiutamente $a_{1}^{\prime}, a_{1}, a_{1,}, a_{2}^{\prime}$.

Gli spostamenti per il sermento $A B$ sono

$$
\begin{gathered}
\xi=\frac{1}{\omega_{1}{ }^{\prime}}\left\{A_{1}{ }^{\prime} J_{1}\left(\omega_{1}{ }^{\prime}\right)+B_{1}{ }^{\prime} Y_{1}\left(\omega_{1}{ }^{\prime}\right)\right\} \sin n\left(t-\tau_{1}{ }^{\prime}\right) \\
\zeta=\frac{h_{1}}{2 a_{1}{ }^{\prime}}\left\{A_{1}^{\prime} J_{0}\left(\omega_{1}{ }^{\prime}\right)+B_{1}{ }^{\prime} Y_{0}\left(\omega_{1}{ }^{\prime}\right)\right\} \sin n\left(t-\tau_{1}{ }^{\prime}\right) \\
\omega_{1}^{\prime}=\frac{2 n a^{\prime}}{i g h_{1}} \sqrt{1-\frac{x}{a_{1}}}
\end{gathered}
$$

La condizione al contorno $A, O$, per $\omega^{\prime}{ }_{1}=O$, i che sia sempre $\zeta=\mathrm{O}$, ciò̀

$$
A_{1}^{\prime} J_{1}(0)+B_{1}^{\prime} Y_{1}(0)=0 \text { da cui } \frac{B_{1}^{\prime}}{A_{1}^{\prime}}=-\frac{J_{1}(0)}{Y_{1}(0)}
$$

Poiché $\lim _{(1)=0} \frac{I_{t}(\omega)}{Y_{1}(\omega)}=0$, consegue $B^{\prime}{ }_{1}=0$. Perciò, per punti situati su AB, abbiamo

$$
\begin{aligned}
& \xi=\frac{A s^{\prime}}{\omega_{1}^{\prime}} J_{1}\left(\omega_{1}{ }^{\prime}\right) \sin n\left(t-\tau_{1}{ }^{\prime}\right) \\
& \zeta=\frac{h_{1}}{2 a_{1}^{\prime}} A_{1}^{\prime} J_{0}\left(\omega_{1}{ }^{\prime}\right) \sin n\left(t-\tau_{1}^{\prime}\right)
\end{aligned}
$$

In $\mathrm{B}$, dove $x=0$, ̀̀

$$
\omega_{i}^{\prime}=\frac{2 n a_{1}^{\prime}}{\sqrt{g h_{1}}}=n \alpha_{1}^{\prime} \quad, \quad \text { essendo } \alpha_{1}^{\prime}=\frac{2 a_{1}^{\prime}}{\gamma^{\prime} g h_{1}}
$$

In $B \grave{e}$ pertanto

$$
\begin{aligned}
& \S=A_{1}^{\prime} \frac{1}{n \cdot \alpha_{1}{ }^{\prime}} J_{1}\left(n \alpha_{1}{ }^{\prime}\right) \sin n\left(t-\tau_{1}{ }^{\prime}\right) \\
& \zeta=A_{1}^{\prime} \frac{h_{1}}{2 a_{1}^{\prime}} J_{0}\left(\alpha_{1}\right) \sin n\left(t-\tau_{1}{ }^{\prime}\right)
\end{aligned}
$$


Analogamente, per il segmento BC abbiamo

$$
\begin{aligned}
& \xi=\frac{1}{\omega_{1}}\left\{A_{1} J_{1}\left(\omega_{1}\right)+B_{1} Y_{1}\left(\omega_{1}\right)\right\} \sin n\left(t-\tau_{1}\right) \\
& \zeta=-\frac{h}{2 a_{1}}\left\{A_{1} J_{0}\left(\omega_{1}\right)+B_{1} Y_{0}\left(\omega_{1}\right)\right\} \sin n\left(t-\tau_{1}\right) \\
& \omega_{1}=\frac{2 n a_{1}}{\sqrt{g h_{1}}} \sqrt{1+\frac{x}{a_{1}}}
\end{aligned}
$$

In $B$, dove $x=0$, posto $(1)_{1}=\frac{2 n c_{1}}{\sqrt{g h_{1}}}=n\left(_{1}\right.$, le equazioni scritte sono quelle degli spostamenti in B. Uguagliandoli a quelli della $\left({ }^{2}\right)$, avremo:

$$
\begin{gathered}
\tau_{1}^{\prime}=\tau_{1}=\tau \\
\frac{1}{\alpha_{1}}\left\{A_{1} J_{1}\left(n \alpha_{1}\right)+B_{1} Y_{1}\left(n \alpha_{1}\right)\right\}=\frac{A_{1}^{\prime}}{\alpha_{1}^{\prime}} J_{1}\left(n \alpha_{1}{ }^{\prime}\right) \\
-\frac{1}{a_{1}}\left\{A_{1} J_{0}\left(n \alpha_{1}\right)+B_{1} Y_{0}\left(n u_{1}\right)\right\}=\frac{1}{a_{1}} A_{1}^{\prime} J_{0}\left(n \alpha_{1}{ }^{\prime}\right)
\end{gathered}
$$

Osserviamo che $\frac{a_{4}}{a_{1}^{\prime}}=\frac{\alpha_{1}}{u_{1}{ }^{\prime}}$. Fatte le posizioni

$$
\begin{aligned}
& \Lambda_{1}=J_{1}\left(n \alpha_{1}\right) Y_{0}\left(n \alpha_{1}\right)-J_{0}\left(n \alpha_{1}\right) Y_{1}\left(n a_{1}\right) \\
& \lambda_{1}=J_{1}\left(n \alpha_{1}^{\prime}\right) Y_{0}\left(n \alpha_{1}\right)+J_{0}\left(n \alpha_{1}^{\prime}\right) Y_{1}\left(n \alpha_{1}\right) \\
& u_{1}=J_{1}\left(n u_{1}\right) J_{0}\left(n a_{1}^{\prime}\right)+J_{1}\left(n a_{1}^{\prime}\right) J_{0}\left(n \alpha_{1}\right)
\end{aligned}
$$

otterremo

$$
A_{1}=\frac{u_{1}}{u_{1}^{\prime}} \frac{\lambda_{1}}{I_{1}} A_{1}^{\prime} \quad, \quad B_{1}=-\frac{a_{1}}{u_{1}^{\prime}} \frac{Z_{1}^{\prime}}{\Delta_{1}} A_{1}^{\prime} .
$$

Per il segmento $\mathrm{BC}$ avremo pertanto

Ponendo

$$
\begin{aligned}
& \Xi=\frac{A_{1}^{\prime}\left(u_{1}\right.}{(1)_{1} \lambda_{1}\left({ }_{1}^{\prime}\right.}\left\{\lambda_{1} J_{1}\left((1)_{1}\right)-H_{1} Y_{1}\left(()_{1}\right)\right\} \sin n(t-\tau) \\
& \zeta=-\frac{h_{1} A_{1}^{\prime}\left(\prime_{1}\right.}{2 a_{1} J_{1} \alpha_{1}^{\prime}}\left\{\lambda _ { 1 } J _ { 0 } \left(\left(u_{1}\right)-u_{1} Y_{0}\left(\left(u_{1}\right)\right\} \sin n(t-r) .\right.\right.
\end{aligned}
$$

$$
\omega_{1}=\frac{2 n a_{1}}{\mid g h_{1}} \sqrt{/ 1-\frac{p_{1}}{a_{1}}}=n \beta_{1}
$$


in queste equazioni, avremo gli spostamenti sul punto $C$; essi sono:

$$
\begin{aligned}
& \xi=\frac{A_{1}{ }^{\prime} \alpha_{1}}{n \beta_{1} \Delta_{1} \alpha_{1}{ }^{\prime}}\left\{\lambda_{1} J_{1}\left(n \beta_{1}\right)-u_{1} Y_{1}\left(n \beta_{1}\right)\right\} \sin n(t-\tau) \\
& \zeta=-\frac{h_{1} A_{1}{ }^{\prime} \alpha_{1}}{2 a_{1} \Delta_{1} \alpha_{1}}\left\{\gamma_{1} J_{0}\left(n \beta_{1}\right)-\mu_{1} Y_{0}\left(n \beta_{1}\right)\right\} \sin n(t-\tau)
\end{aligned}
$$

Per DC

$$
\begin{aligned}
& \xi=\frac{1}{\omega_{2}}\left\{A_{2} J_{1}\left(\omega_{2}\right)+B_{2} Y_{1}\left(\omega_{2}\right)\right\} \sin n\left(t-\mathrm{T}_{2}\right) \\
& \zeta=\frac{h_{2}}{2 a_{2}}\left\{A_{2} J_{0}\left(\omega_{2}\right)+B_{2} Y_{\mathrm{o}}\left(\omega_{2}\right)\right\} \sin n\left(t-\mathrm{\tau}_{2}\right) \\
& \omega_{2}=\frac{2 n a_{2}}{\sqrt{g h_{2}}} \sqrt{1-\frac{x}{a_{2}}}
\end{aligned}
$$

In $D$, dove $x=0, \omega_{2}=\frac{2 n r_{2}}{\sqrt{g} h_{2}}=n \alpha_{2}$ gli spostamenti divengono

$$
\begin{aligned}
& \xi=\frac{1}{n \alpha_{2}}\left\{A_{2} J_{1}\left(n \alpha_{2}\right)+B_{2} Y_{1}\left(n \alpha_{2}\right)\right\} \sin n\left(t-\tau_{2}\right) \\
& \zeta=\frac{h_{2}}{2 a_{2}}\left\{A_{2} J_{0}\left(n \alpha_{2}\right)+B_{2} Y_{0}\left(n \alpha_{2}\right)\right\} \sin n\left(t-\tau_{2}\right)
\end{aligned}
$$

Per il tratto DE

$$
\begin{aligned}
& \xi=\frac{1}{\left(_{2}^{\prime}{ }^{\prime}\right.}\left\{A_{2}^{\prime} J_{1}\left(\omega_{2}{ }^{\prime}\right)+B_{2}{ }^{\prime} Y_{1}\left(\omega_{2}{ }^{\prime}\right)\right\} \sin n\left(t-\mathrm{r}_{2}{ }^{\prime}\right) \\
& \zeta=-\frac{h_{2}}{2 a_{2}{ }^{\prime}}\left\{A_{2}^{\prime} J_{0}\left(\omega_{2}{ }^{\prime}\right)+B_{2}{ }^{\prime} Y_{0}\left(\omega_{2}{ }^{\prime}\right)\right\} \sin n\left(t-r_{2}{ }^{\prime}\right) \\
& \omega_{2}^{\prime}=\frac{2 n a_{2}^{\prime}}{\sqrt{g h_{2}}} \sqrt{1+\frac{x}{a_{2}{ }^{\prime}}}
\end{aligned}
$$

In $\mathrm{E}$, per $\omega_{2}^{\prime}=0$, ̀̀ $\xi=0$, ciò̀

$$
A_{2}{ }^{\prime} J_{1}(0)+B_{2}{ }^{\prime} Y_{1}(0)=0
$$

Per una proprietà delle funzioni di Bessel, già richiamata, consegue $\mathrm{B}_{2}^{\prime}=\mathrm{O}$. Per DE è pertanto 


$$
\begin{aligned}
& \xi=\frac{A_{2}^{\prime}}{\omega_{2}{ }^{\prime}} J_{1}\left(\omega_{2}{ }^{\prime}\right) \sin n\left(t-\tau_{2}{ }^{\prime}\right) \\
& \zeta=-\frac{h_{2}}{2 a_{9}{ }^{\prime}} A_{2}{ }^{\prime} J_{\mathrm{o}}\left(\omega_{2}{ }^{\prime}\right) \sin n\left(t-\tau_{2}{ }^{\prime}\right)
\end{aligned}
$$

In $\mathbf{D} \dot{\mathrm{e}}$

In $D$ i pertanto

$$
\omega_{2}{ }^{\prime}=\frac{2 n \boldsymbol{a}_{2}{ }^{\prime}}{\sqrt{g h_{\mathrm{2}}}}=n^{\cdot}{u_{2}}^{\prime} \quad u_{2}{ }^{\prime}=\frac{2 \boldsymbol{a}_{2}{ }^{\prime}}{\sqrt{g h_{2}}}
$$

$$
\begin{aligned}
& \xi=\frac{A_{2}{ }^{\prime}}{n \alpha_{2}{ }^{\prime}} J_{1}\left(n \alpha_{2}{ }^{\prime}\right) \sin n\left(t-\tau_{2}{ }^{\prime}\right) \\
& \zeta=-\frac{h_{2}}{2 a_{2}{ }^{\prime}} A_{0}{ }^{\prime} J_{0}\left(n \alpha_{2}{ }^{\prime}\right) \sin n\left(t-\tau_{2}{ }^{\prime}\right) .
\end{aligned}
$$

Uguagliando la [9] alle [10], risulta

$$
\begin{gathered}
\tau_{0}=\tau_{2}^{\prime}=\tau \\
\frac{1}{\alpha_{2}}\left\{A_{2} J_{1}\left(n \alpha_{2}\right)+B_{2} Y_{1}\left(n \sigma_{2}\right)\right\}=\frac{A_{2}^{\prime}}{\alpha_{2}^{\prime}} J_{1}\left(n \alpha_{2}^{\prime}\right) \\
\frac{1}{a_{2}}\left\{A_{2} J_{0}\left(n \alpha_{2}\right)+B_{2} Y_{0}\left(n \alpha_{2}\right)\right\}=-\frac{A_{2}^{\prime}}{a_{2}^{\prime}} J_{0}\left(n \alpha_{2}{ }^{\prime}\right)
\end{gathered}
$$

Poiché $\frac{\alpha_{2}}{\alpha_{a}{ }^{\prime}}=\frac{a_{2}}{a_{o}{ }^{\prime}}$, fatte le posizioni

$$
\begin{aligned}
& \Delta_{2}=J_{1}\left(n \alpha_{2}\right) Y_{0}\left(n \alpha_{2}\right)-J_{0}\left(n \alpha_{2}\right) Y_{1}\left(n \alpha_{2}\right) \\
& \lambda_{2}=J_{1}\left(n \alpha_{2}^{\prime}\right) Y_{0}\left(n \alpha_{2}\right)+J_{0}\left(n \alpha_{2}^{\prime}\right) Y_{1}\left(n \alpha_{2}\right) \\
& \mu_{2}=J_{1}\left(n \alpha_{2}\right) J_{0}\left(n \alpha_{2}{ }^{\prime}\right)+J_{0}\left(n \alpha_{2}\right) J_{1}\left(n \alpha_{2}{ }^{\prime}\right)
\end{aligned}
$$

si ottiene

$$
\begin{aligned}
& A_{2}-\frac{\alpha_{2}}{\alpha_{2}^{\prime}} \frac{\lambda_{2}}{\Delta_{2}} A_{z}^{\prime}, \\
& B_{2}=-\frac{\alpha_{2}}{\alpha_{2}^{\prime}} \frac{\mu_{2}}{\Delta_{n}} A_{2}^{\prime} .
\end{aligned}
$$

Per il segmento DC è

$$
\begin{aligned}
& \xi=\frac{1}{\omega_{0}} \frac{\alpha_{2}}{\alpha_{9}^{\prime}} \frac{A_{2}^{\prime}}{\Delta_{2}}\left\{j_{2} j_{1}\left(\omega_{2}\right)-\mu_{2} Y_{1}\left(\omega_{2}\right)\right\} \sin n(t-\tau) \\
& \zeta=\frac{h_{2}}{2 a_{2}} \frac{\alpha_{2}}{\alpha_{2}^{\prime}} \frac{A_{2}^{\prime}}{\Delta_{2}}\left\{\lambda_{2} J_{\|}\left(\omega_{2}\right)-\mu_{2} Y_{0}\left(\omega_{2}\right)\right\} \sin n(\tau-t)
\end{aligned}
$$


Poiché in $\mathrm{C}$ è

$$
\omega_{2}=\frac{2 n a_{2}}{\sqrt{g h_{2}}} \sqrt{1-\frac{p_{2}}{a_{2}}}=n \beta_{2} ;
$$

gli spostamenti in C hanno l'espressione

$$
\left\{\begin{array}{l}
\xi=\frac{\alpha_{2} A_{2}^{\prime}}{\sigma_{2}{ }^{\prime} n \beta_{2} \Delta_{2}}\left\{\lambda_{2} J_{1}\left(2 \beta_{2}\right)-\mu_{2} Y_{1}\left(n \beta_{2}\right)\right\} \sin n(t-\tau) \\
\zeta=\frac{h_{2}}{2 a_{2}} \frac{\alpha_{2}}{\alpha_{2}{ }^{\prime}} \frac{A_{2}{ }_{2}}{\Delta_{2}}\left\{\lambda_{2} J_{0}\left(n \beta_{2}\right)-\mu_{2} Y_{0}\left(n \beta_{2}\right)\right\} \sin n(t-\tau) .
\end{array}\right.
$$

Uguagliando le [8] alle [1 $\left.4^{\prime}\right]$ si perviene alla sequente equazione per i periodi:

$$
\begin{aligned}
& \left.\left.\frac{h_{1} \beta_{1} a_{2}}{h_{2} \beta_{2} a_{1}}\right\},_{1} J_{0}\left(n \beta_{1}\right)-n_{1} V_{0}\left(n \beta_{1}\right)\right\}\left\{, J_{2}\left(n \beta_{z}\right)-H_{2} Y_{1}\left(n \beta_{z}\right)\right\}+ \\
& \quad+\left\{\lambda_{1} J_{1}\left(n \beta_{1}\right)-\mu_{1} Y_{1}\left(n \beta_{1}\right)\right\} \cdot\left\{\lambda_{2} J_{0}\left(n \beta_{2}\right)-\mu_{2} Y_{0}\left(n \beta_{2}\right)\right\}=0
\end{aligned}
$$

dove $\lambda_{1}, \mu_{1}, \lambda_{2}, \mu_{2}$ hanno le espressioni riportate nelle [4], [5], [12], [13] rispettivamente $(*)$.

2. - Applichiamo la teoria esposta al lago di Scanno.

La tabella I riporta i dati tratti dalla forma e dalla batimetria del lago, dati che hanno servito, fra l'altro, a determinare la curva normale, come da fig. 2 .

Per l'applicazione della teoria esposta al $\mathrm{n}$. 1, la curra normale è stata schematizzata in quattro tratti raccordati (fig. 00).

Valendoci dei valori riportati nella tab. I abbiamo determinato le equazioni delle rette cui appartengono i tratti $1,2,3,4$, della figr. 1 .

(*) I giapponesi Nakamura e Honda, trattando un problema analogo (3), incorsero in due errori: uno dovuto ad una svista di Chrystal - contenuta nella prima redazione della sua memoria (2) - l'altro -- più grave - conseguente ad incomprensibili dimenticanze di segno nel corso della trattazione analitira. Questo fatto, principalmente. condusse gli autori citati ad ottenere per l'uninodale del lago di Hakoné un valore di $22 m, 47$, mentre le osservazioni dawano soltanto $15^{\text {"n }}$ "a.

Tale divergenza, per loro inesplicabile, venne attribuita alla scarsa approssimazione del metodo. In realtà, corretti gli errori di segno arcennati e rifatti i calcoli con la formula da noi ottenuta, si ottiene per l'uninodale del lago Hakoné il valore di $1 t^{\mathrm{m}}$ ca, molto prossimo quindi a quello osservato. 


\section{Tabella I}

\begin{tabular}{|c|c|c|c|c|c|}
\hline שัٌ & $\mathbf{x}$ & $\begin{array}{l}S(\mathrm{x}) \\
\mathrm{m}^{2}\end{array}$ & $\begin{array}{c}b(x) \\
m\end{array}$ & 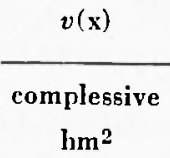 & $\begin{array}{l}\sigma(\mathrm{x}) \\
\mathrm{hm}^{3}\end{array}$ \\
\hline 0 & 0 & 0 & 0 & 0 & 0 \\
\hline 1 & 80 & 6510 & 421 & 3,4210 & 2,77296 \\
\hline 2 & 160 & 9060 & 492 & 6,9760 & 4,45752 \\
\hline 3 & 240 & 11080 & 572 & 11,4560 & 6,33776 \\
\hline 4 & 320 & 13320 & 662 & 16,2880 & 8,81784 \\
\hline 5 & 400 & 15960 & 724 & 21,6640 & 11,55504 \\
\hline 6 & 480 & 17560 & 736 & 27,4240 & 12,92416 \\
\hline 7 & 560 & 18660 & 758 & 33,2480 & 14,14428 \\
\hline 8 & 640 & 18080 & 750 & 39,2000 & 13,56000 \\
\hline 9 & 720 & 17940 & 714 & 45,0880 & 12,80916 \\
\hline 10 & 800 & 17640 & 680 & 50,7200 & 11,99520 \\
\hline 11 & 880 & 15760 & 650 & 56,0000 & 10,24400 \\
\hline 12 & 960 & 15960 & 712 & 61,3760 & 11,36352 \\
\hline 13 & 1040 & 17120 & 736 & 67,0720 & 12,60032 \\
\hline 14 & 1120 & 17800 & 748 & 73,8880 & 13,31440 \\
\hline 15 & 1200 & 18320 & 776 & 80,8320 & 14,21632 \\
\hline 16 & 1240 & 17040 & 800 & 84,5120 & 13,63200 \\
\hline 17 & 1280 & 11120 & 726 & 88,5440 & 10,25112 \\
\hline 18 & 1360 & 11840 & 520 & 91,6560 & 6,15680 \\
\hline 19 & 140 & 10200 & 472 & 98,8800 & 4,81440 \\
\hline 20 & 1520 & 6520 & 368 & 102,3040 & 2,39936 \\
\hline 21 & 1600 & 5360 & 348 & 105,2800 & 1,86528 \\
\hline 22 & 1680 & 5060 & 396 & 108,0640 & 2,00376 \\
\hline 23 & 1760 & 2600 & 380 & 111,1040 & 0,98800 \\
\hline 24 & 1814 & 0 & 0 & 112.5120 & 0 \\
\hline
\end{tabular}


Ciò ci ha consentito di calcolare i valori delle costanti $h_{1}, h_{.,}$, $h$... Si è ottenuto:

$a_{1}{ }^{\prime}=p_{1}{ }^{\prime}=30,97 \quad ; \quad a_{1}=90,78 \quad ; \quad a_{2}=89,84 ; a_{\mathrm{a}}{ }^{\prime}=p_{2}{ }^{\prime}=33,14 h \cdot h^{*} \quad ;$ $p_{1}=25,81 ; p_{2}=22,60 \mathrm{hm}^{2}$

$h_{1}=14,82 ; h_{\mathrm{g}}=14,17 ; h=10,60 \mathrm{hm}^{3}$

$\mathrm{Ne}$ consegue:

$$
\begin{aligned}
& \alpha_{1}=150,93 ; \alpha_{2}=152,41 \quad \alpha_{1}^{\prime}=51,38 ; \alpha_{2}{ }^{\prime}=56,22 \mathrm{sec} ; \\
& \beta_{1}=127,69 ; \beta_{0}=131,87 \text { sec. }
\end{aligned}
$$

Periodi. - I valori ottenuti consentono la risoluzione dell'equa-

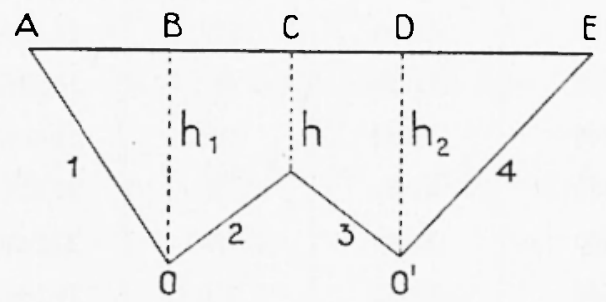

Fig. 1

zione dei periodi delle possibili oscillazioni libere del layo di Scanno.

Per quanto riguarda la sessa uninodale, il valore di $n$ che rende praticamente nulla la [15] è .02936 , cui corrisponde per il periodo dell'uninodale il valore

$$
T_{1}=214^{*} \text {. }
$$

La sessa binodale corrisponde al valore $n=.0528$, che annulla la $[15]$ :

consegue

$$
T_{\mathrm{2}}=119^{*}
$$

La terza radice della [15], nell'ordine, si ha per $n=.0706$, cui corrisponde

$$
T_{3}=89^{\star}
$$

periodo della sessa trinodale.

Nodi. - E facile provare che l'uninodo cade nel segmento BC. 
Nel nodo dev'essere, naturalmente, $\zeta=0$. Con riferimento alla $2^{\text {a }}$ delle [7], dev'essere cioè

$$
\frac{Y_{0}\left(\omega_{1}\right)}{J_{0}\left(\omega_{1}\right)}=-\frac{\lambda_{1}}{\mu_{1}}
$$

Ora, per l'oscillazione libera uninodale, è $\lambda_{1}=+0.06471$; $u_{1}=-0.29516 ;$ in corrispondenza del nodo, dovrà essere pertanto

$$
\frac{Y_{0}\left(\omega_{1}\right)}{J_{0}\left(\omega_{1}\right)}=-0,21923
$$

Ciò si verifica per

Ma

$$
\omega_{1}=3,74355 \text {. }
$$

da cui

$$
\omega_{1}=n \alpha_{1} \sqrt{1+\frac{x}{a_{1}}}
$$

$$
x=-a_{1}\left[1-\left(\frac{\omega_{1} T}{2 \pi \alpha_{1}}\right)^{2}\right]
$$

Ricordando i valori di $a_{1}$ e $\alpha_{1}$ e posto $\mathrm{T}=214^{a}$ (periodo dell'uninodale), consegue

$$
x=-25,99,
$$

ascissa dell'uninodo con riferimento al sistema di assi cartesiani con origine in $\mathrm{B}$ ( $\mathrm{fig}$. 1 ).

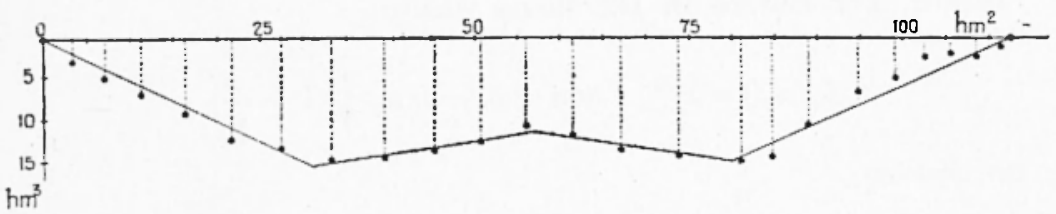

Fig. 2

L'ascissa dell'uninodo in unità delle $v(x)$ - vedi tabella - sarà allora, tenendo conto del valore di $a_{1}^{\prime}$,

$$
v\left(x_{1}\right)=56,96 \mathrm{lim}^{2} \text {. }
$$

L'uninodo dista quindi dall'estremo-origine m 894.

Affinché un binodo sia nel tratto $\mathbf{A B}$, dovrà essere $J_{0}\left(\omega_{1}{ }^{\prime}\right)=0$. Poiché

$$
\omega_{1}^{\prime}=n \alpha_{1}^{\prime} \sqrt{1-\frac{x}{a_{1}^{\prime}}},
$$


dovrà essere

$$
x=a_{i}^{\prime}\left[1-\left(\frac{\omega_{1}^{\prime} T}{2 \pi \alpha_{1}^{\prime \prime}}\right)^{2}\right]
$$

Un binodo sarà in $\mathrm{AB}$ se $\omega_{1}{ }^{\prime}<n \alpha_{1}{ }^{\prime}$.

Per la hinodale $\left(\mathrm{T}=119^{\mathrm{s}}\right) \grave{\mathrm{e}}$

$$
n u_{1}^{\prime}=2,713 .
$$

Poiché le prime radici di $J_{0}(\omega)=0$ sono

$$
2,4048 ; 5,5201 ; 8,6537 ; \ldots
$$

e poiché la prima di tali radici è appunto minore di $n \alpha_{2}^{\prime}$, se ne conclude un che un binodo è effettivamente nel tratto $A B$. La sua ascissa si deduce dalla [16], dove va fatto $\left({ }^{\prime}\right)^{\prime}=2,4048 ; \mathrm{T}=119$ sec. Risulta

$$
x=6,6307 \text {. }
$$

In unità delle $v(x)$, date dalla tabella, avremo

$$
v\left(x_{2}{ }^{2}\right)=30,97-6,6307=24,3393 \mathrm{hm}^{2},
$$

valore delll'ascissa del primo binodo, nel sistema di ordinate, cui ̀̀ riferita la curva normale.

La distanza del I binodo dall'estremo-origine è quindi di m 437 .

Il secondo binodo cade in DE, come è facile provare.

Infatti, per cadere in $\mathrm{DE}$ dovrà essere

$$
J_{0}\left(\omega_{0}{ }^{\prime}\right)=0, \quad \text { con } \omega_{2}{ }^{\prime}=n \alpha_{2}{ }^{\prime} \sqrt{1+\frac{x}{a_{2}{ }^{\prime}}}
$$

Se ne deduce

$$
x=-a_{g^{\prime}}\left[1-\left(\frac{(1)_{g}^{\prime} T}{2 \pi u_{\underline{g}}{ }^{\prime}}\right)^{2}\right]
$$

Dovrà essere quindi $\omega_{9}{ }^{\prime}<n \alpha_{2}{ }^{\prime}$, ciò che in effetti si verifica, essendo $n \alpha_{2}{ }^{\prime}=2,968$. si ha

Posto nella [17] $a_{2}^{\prime}=33,14 ; \quad \omega_{2}^{\prime}=2,4048 ; \mathrm{T}=119^{\mathrm{s}} ; \omega_{2}^{\prime}=56,222$,

$$
x=-11,3902 \text {. }
$$

L'ascissa del secondo binodo è pertanto - nella successione delle $v(x)$ riportate nella tabella $\mathrm{I}$,

$$
v\left(x_{n^{\prime \prime}}\right)=79,3731+11,3902=90,7633 \mathrm{hm}^{2}
$$


Il II binodo dista quindi dall'estremo-origine m. 1309. Passiamo ai trinodi.

Per avere un trinodo in $\mathrm{AB}$, dovrà essere $J_{0}\left(\left(_{1}{ }_{1}{ }^{\prime}\right)=0\right.$, e una radice di questa equazione risultare minore di $n \alpha_{1}^{\prime}$. Per la trinodale è

$$
n\left(\iota_{1}^{\prime}=3,6271\right. \text {. }
$$

Un trinodo quindi $\grave{e}$ sicuramente nel tratto $\mathrm{AB}$. Vale perciò la

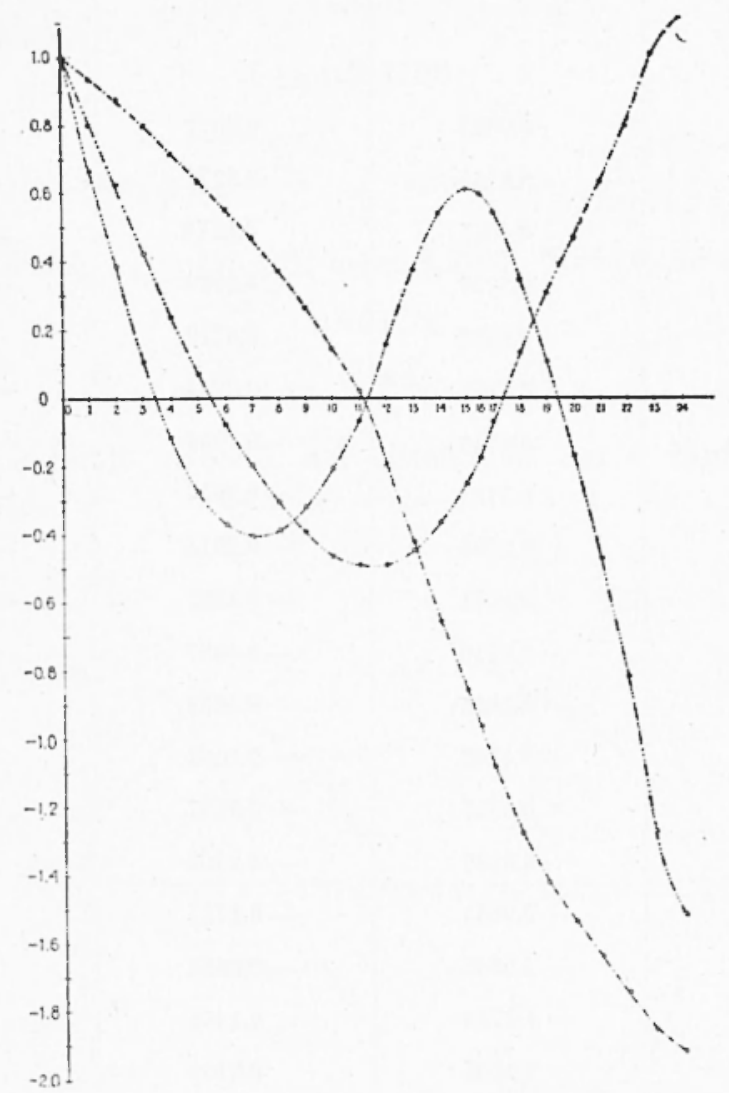

Fig. 3

[16]; dalla quale si deduce - fatto $\left({ }^{\prime}{ }_{1}^{\prime}=2,4048 ; T=89^{\text {s }} ; a_{1}{ }^{\prime}=30,97\right.$

$$
x=17,3556 \text {. }
$$

Nella serie di valori progressivi delle $v(x)$, l'ascissa del primo trinodo è allora

$$
v\left(x_{3}{ }^{1}\right)=13,61,
$$

e la sua distanza dall'estremo-origine vale m. 276 . 
Tabetua II - Andamento degli spostamenti verticali

\begin{tabular}{|c|c|c|c|}
\hline Sezioni & Üninodale & Binodale & Trinodale \\
\hline 0 & 1 & 1 & 1 \\
\hline 1 & 0.9383 & 0.8071 & 0.6691 \\
\hline 2 & 0.8762 & 0.6274 & 0.3870 \\
\hline 3 & 0.8005 & 0.4271 & 0.1076 \\
\hline 4 & 0.7229 & 0.2429 & -0.1108 \\
\hline 5 & 0.6403 & 0.0729 & -0.2707 \\
\hline 6 & 0.5565 & $-0.07+4$ & -0.3666 \\
\hline 7 & 0.4740 & -0.1933 & --0.4021 \\
\hline 8 & 0.3781 & -0.3017 & -0.3864 \\
\hline 9 & 0.2694 & -0.3916 & -0.3193 \\
\hline 10 & 0.1471 & -0.4582 & -0.2031 \\
\hline 11 & 0.0240 & -0.4897 & -0.0635 \\
\hline 12 & -0.1926 & -0.4883 & 0.1606 \\
\hline 13 & -0.4192 & $-0.4+98$ & 0.3750 \\
\hline 14 & -0.6557 & -0.3687 & $0.5+56$ \\
\hline 15 & -0.8589 & -0.2502 & 0.6110 \\
\hline 16 & -0.9654 & -0.1717 & 0.6018 \\
\hline 17 & -1.0865 & -0.0664 & 0.5435 \\
\hline 18 & -1.2783 & 0.1354 & 0.3483 \\
\hline 19 & -1.4205 & 0.3109 & 0.1064 \\
\hline 20 & -1.5392 & 0.4749 & -0.1653 \\
\hline 21 & -1.6467 & 0.6376 & -0.4701 \\
\hline 22 & -1.7505 & 0.8060 & -0.8178 \\
\hline 23 & --1.8667 & 1.0084 & -1.2765 \\
\hline 24 & -1.9219 & 1.1099 & -1.5204 \\
\hline
\end{tabular}




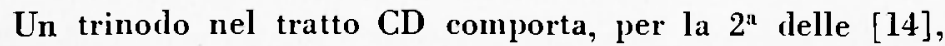

da cui

$$
\lambda_{2} J_{0}\left(\omega_{2}\right)-\mu_{2} Y_{0}\left(\omega_{0}\right)=0 \text {, }
$$

$$
\frac{J_{0}\left(\omega_{2}\right)}{Y_{0}\left(\omega_{2}\right)}=\frac{\mu_{2}}{\lambda_{2}} \text {. }
$$

Poiché, per la trinodale, ’̀ $\lambda_{2}=-.07435 ; u_{2}=+.06485$, consegue ciò che richiedle

$$
J_{0}\left(\omega_{2}\right)=-.87357 \cdot Y_{0}\left(\omega_{2}\right) \text {, }
$$

$\mathrm{Ma}$

$$
\omega_{2}=9,3708 \text {. }
$$

$$
\omega_{2}=n \alpha_{2} \sqrt{1-\frac{x}{a_{2}}} \quad, \quad x=a_{2}\left[1-\left(\frac{\omega_{2} T}{2 \pi \alpha_{1}}\right)^{2}\right] .
$$

Fatto $a_{2}=89,84, \alpha_{2}=152,4.13, \omega_{2}=9,3708, \mathrm{~T}=89$, si ottiene

Si ha allora

$$
x=21,70
$$

$$
v\left(x_{3}{ }^{11}\right)=79,37-21,70=57.67 \mathrm{hm}^{2},
$$

ascissa del secondo trinodo, nel sistema in cui è rappresentata la curva normale.

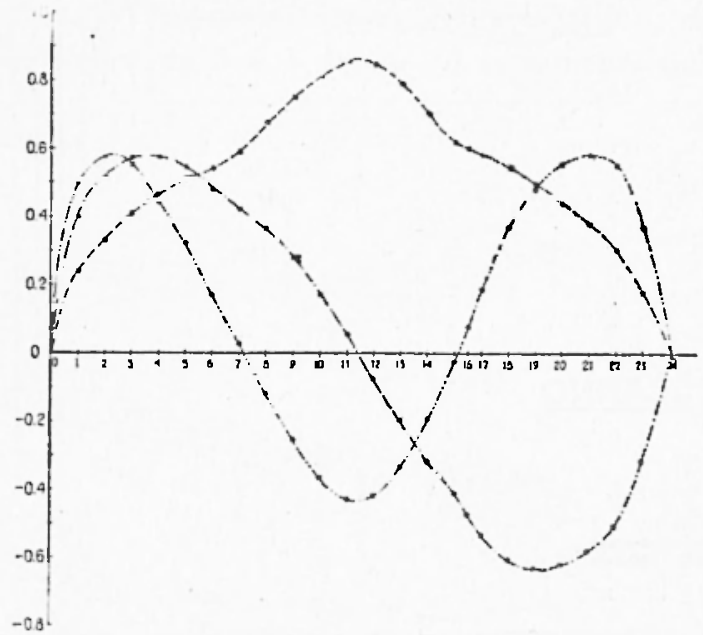

Fig. 4

Il II trinodo dista pertanto dall'estremità-base m 905.

Un trinodo nel quarto tratto $\mathrm{DE}$, presuppone

$$
J_{\mathbf{o}}\left(\omega_{\underline{\alpha}}{ }^{\prime}\right)=0 \text {, }
$$

con una radice minore di $n \alpha_{2}{ }^{\prime}$. E poiché per la trinodale è $n c^{\prime}=3,969$, il terzo trinodo è certamente nel tratto DE. 
Per la $[17]$, dove va fatto $\omega_{2}{ }^{\prime}=2,4048 ; \mathrm{T}=89^{\mathrm{g}} ; \alpha_{2}^{\prime}=56,222$ e $a_{2}{ }^{\prime}=33,14$, si ha

$$
x=-20,974 \text {. }
$$

L'ascissa del terzo trinodo, nel sistema rappresentativo della curva normale, è allora

$$
v\left(x_{\mathrm{s}}{ }^{11}\right)=100,34 \mathrm{hm}^{2}
$$

e la distanza dall'estremità-origine è pari a m 1474 .

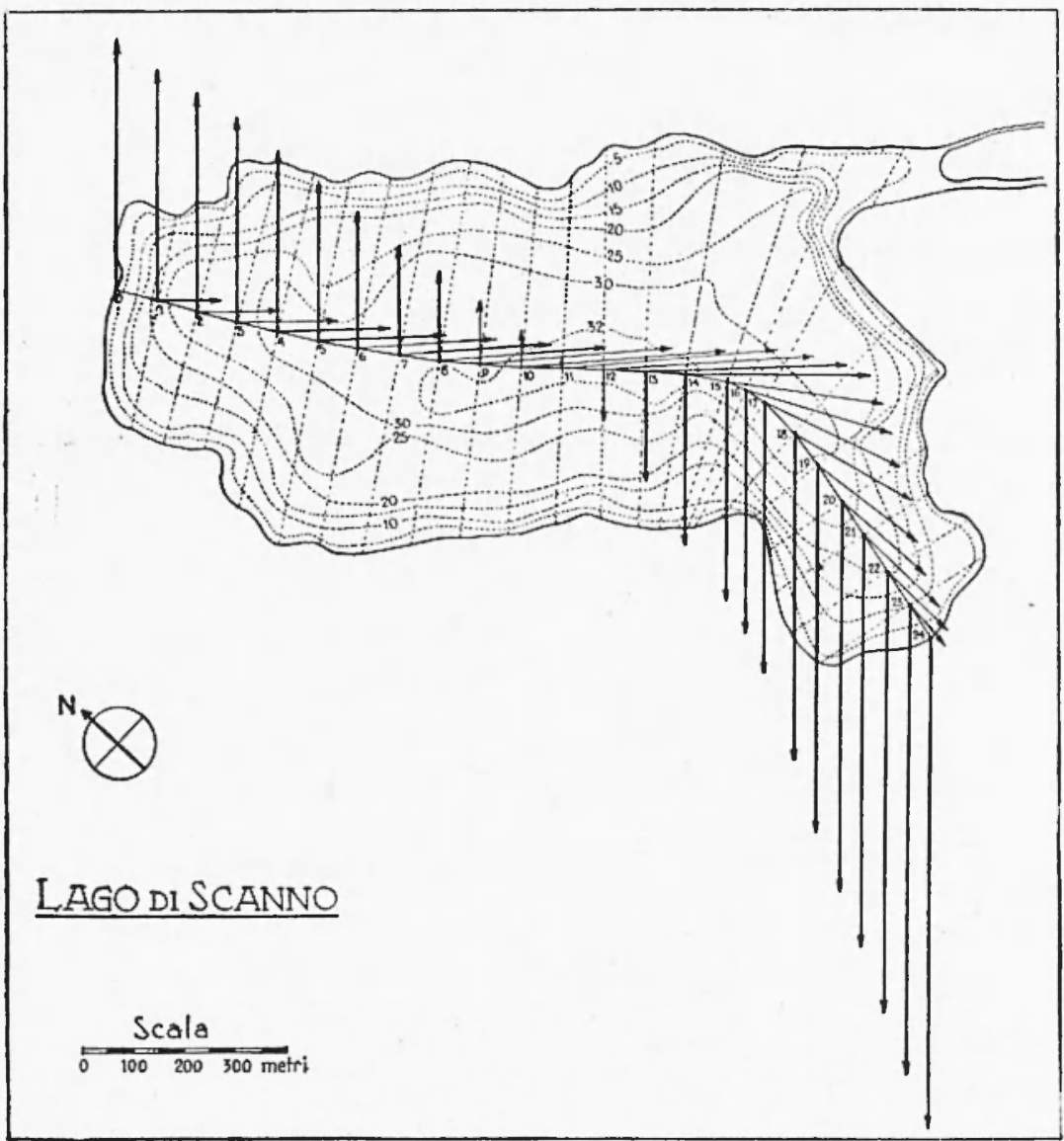

Fig. 5 - Spostamenti relativi verticali e orizzontali per la sessa uninodale (le direzioni degli spostamenti orizzontali sono state un pò deviate alln scopo di evitare sovrapposizioni).

Spostamenti verticali. - I valori relativi degli spostamenti verticali lungo la sezione longitudinale del lago, si possono ottenere, in corrispondenza dei quattro tratti in cui la curva normale è stata suddivisa, valendoci delle equazioni seconde nelle [1], [7], [14] e [10]. 
I valori sono riferiti ad uno spostamento della sezione $\mathrm{O}$ uguale a 1 .

La continuità tra $\mathrm{i}$ valori ottenuti per le sezioni comprese nel tratto $\mathrm{AB}$ e quelli relativi alle sezioni comprese in $\mathrm{BC}$ si ottiene moltiplicando $i$ valori che si traggono dall'espressione in parentesi quadra nella $2^{\mathrm{a}}$ delle [7] per il fattore $-\frac{1}{\Delta_{1}}$.

La continuità fra i valori di BC e quelli compresi in CD si trae dai valori che $\gamma_{2} J_{0}\left(\omega_{2}\right)-\mu_{2} Y_{0}\left(\omega_{2}\right)-$ vedi $2^{\text {a }}$ delle [14] - assume nelle sezioni comprese in $\mathrm{CD}$, moltiplicandoli per il fattore

$$
\frac{a_{1}^{\prime}}{a_{2}{ }^{\prime}} \frac{h_{2}}{h_{1} A_{2}^{\prime} A_{1}^{\prime}} \frac{1}{\Delta_{2}}=-\frac{1}{\Delta_{1} \gamma_{2} J_{0}\left(n \beta_{2}\right)-\mu_{2} Y_{0}\left(n \beta_{2}\right)}=k .
$$

Infine, la continuità fra $\mathrm{CD}$ e $\mathrm{DE}$ è assicurata moltiplicando i valori di - $J_{\mathrm{o}}\left(\omega_{2}\right)$ [formula $\left.(10)\right]$, relativi alle sezioni che entrano in $\mathrm{DE}$, per il fattore

dove $k$ è dato dalla [18].

$$
k \cdot\lrcorner_{2}
$$

Tenendo conto delle precedenti osservazioni, sono stati calcolati gli spostamenti relativi per le sesse uni, -hi- e trinodali, riportati nella tabella II. Le figure da 3 a 7 danno gli andamenti corrispondenti

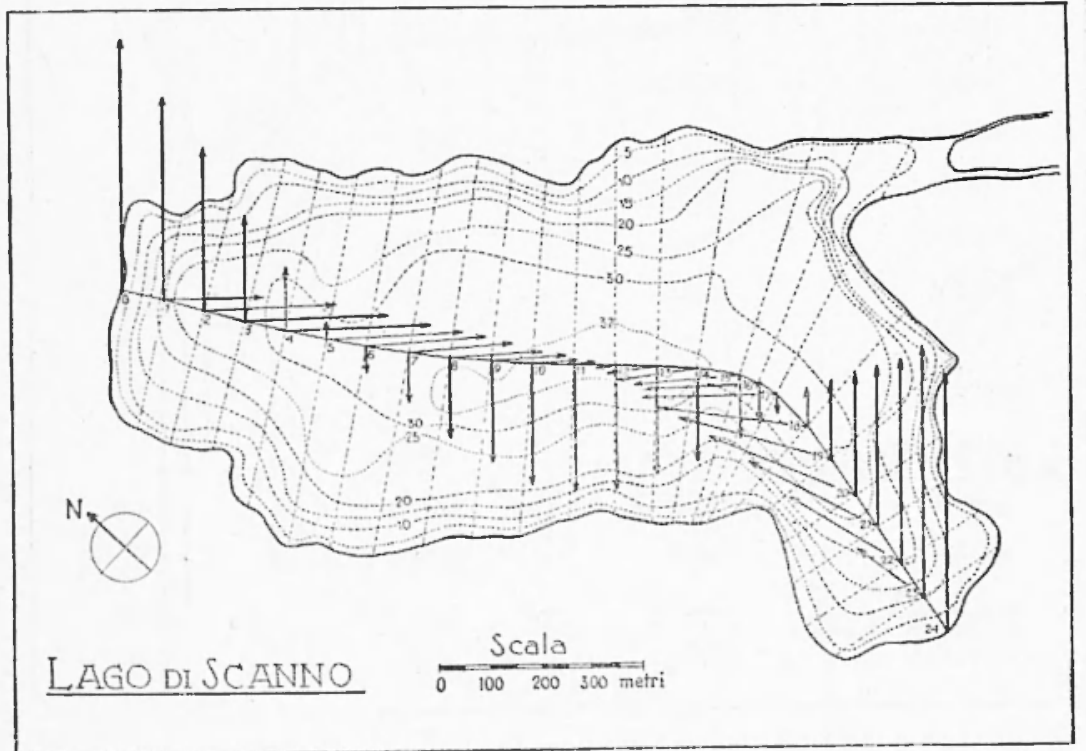

Fig. 6 - Spostamenti relativi verticali e orizzontali per la sessa binodale (i vettori rappresentativi degli spostamenti orizzontali sono stati un pò deviati, per evitare sovrapposizioni). 
Spostamenti orizzontali. - I valori relativi degli spostamenti orizzontali interessanti i punti contenuti nella sezione longitudinale del lago, si possono ottenere facendo uso delle equazioni prime nelle $[1],[7],[14]$ e [10].

Nel tratto AB furono calcolati i valori di $\frac{m}{A_{1}^{\prime}}, \underline{\xi}$.

Per la continuità con $i$ valori relativi al tratto precedente $i$ valori calcolati per $\lambda_{1} J_{1}\left(\omega_{1}\right)-\mu_{1} Y_{1}\left(\omega_{1}\right)$ relativi alle sezioni trasversali contenute nel tratto $\mathrm{BC}$ - equaz. [7] — vanno moltiplicati per il fattore $\frac{1}{\Delta_{1}}$.

Il collegamento fra $\mathrm{i}$ tratti $\mathrm{BC}$ e $\mathrm{CD}$ si ottiene moltiplicando $\mathrm{i}$

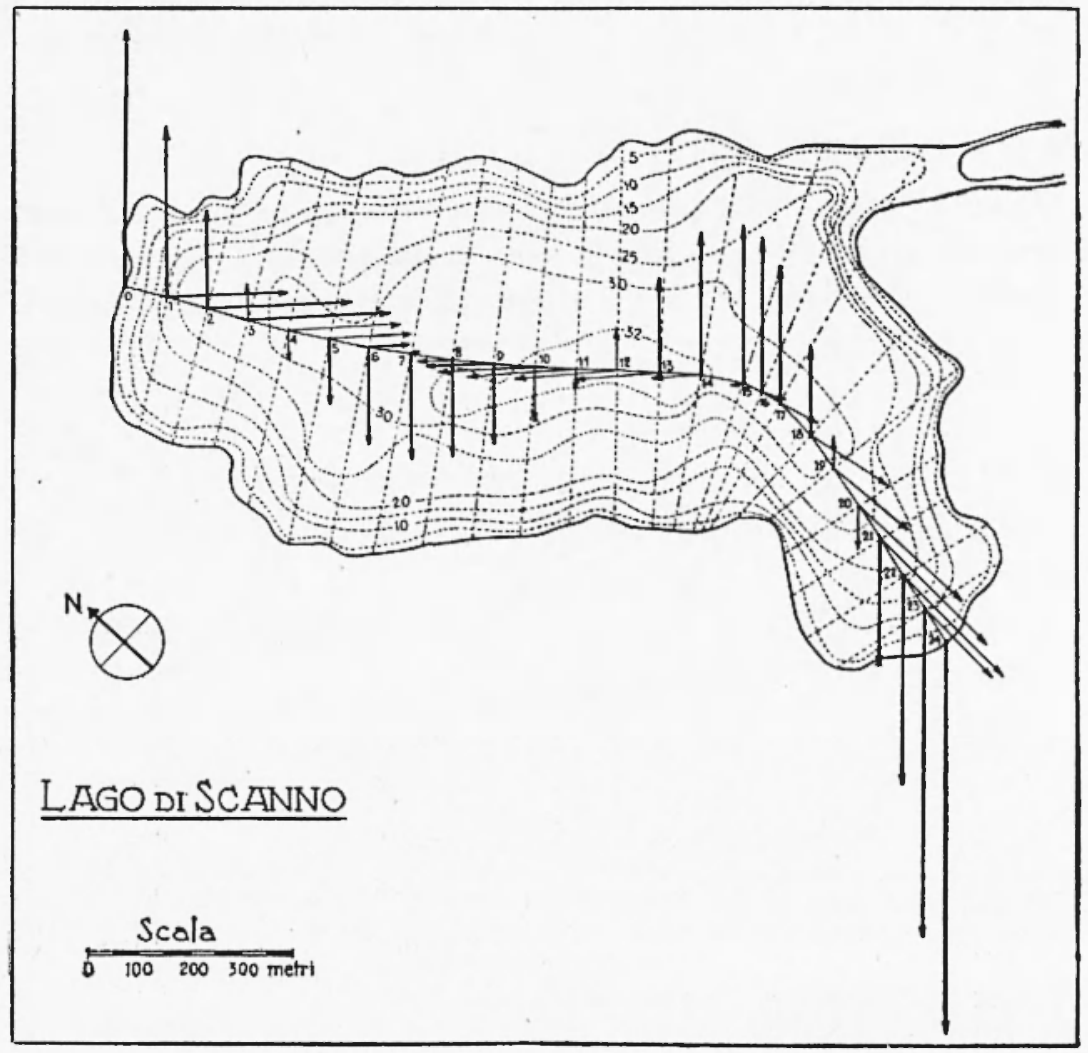

Fig. 7 - Spostamenti relativi verticali e orizzontali per la sessa trinodale (la direzione dei vettori rappresentativi dei movimenti orizzontali è stata un pò mutata, per evitare sovrapposizioni). 
TaBella III - Andamento degli spostamenti orizzontali

\begin{tabular}{|c|c|c|c|}
\hline Sezioni & Uninodale & Binodale & Trinodale \\
\hline 0 & 0 & 0 & 0 \\
\hline 1 & 0.2427 & 0.4055 & 0.4993 \\
\hline 2 & 0.3351 & 0.5189 & 0.5787 \\
\hline 3 & 0.4119 & 0.5742 & 0.5553 \\
\hline 4 & 0.4689 & 0.5787 & 0.4624 \\
\hline 5 & 0.5132 & 0.5454 & 0.3270 \\
\hline 6 & 0.5453 & 0.4835 & 0.1738 \\
\hline 7 & 0.5951 & 0.4253 & 0.0303 \\
\hline 8 & 0.6813 & 0.3724 & -0.1164 \\
\hline 9 & 0.7566 & 0.2901 & -0.2523 \\
\hline 10 & 0.8198 & 0.1799 & -0.3631 \\
\hline 11 & 0.8644 & 0.0592 & -0.4258 \\
\hline 12 & 0.8537 & -0.0699 & -0.4143 \\
\hline 13 & 0.7948 & -0.1948 & -0.3320 \\
\hline 14 & 0.7081 & -0.3133 & -0.1873 \\
\hline 15 & 0.6252 & -0.4059 & -0.0203 \\
\hline 16 & 0.6132 & -0.4693 & 0.0765 \\
\hline 17 & 0.5936 & -0.5327 & 0.1917 \\
\hline 18 & 0.5486 & -0.6058 & 0.3739 \\
\hline 19 & 0.5017 & -0.6300 & 0.4899 \\
\hline 20 & 0.4504 & -0.6224 & 0.5602 \\
\hline 21 & 0.3913 & -0.5848 & 0.5866 \\
\hline 22 & 0.3156 & -0.5061 & 0.5554 \\
\hline 23 & 0.1834 & -0.3165 & 0.3788 \\
\hline 24 & 0 & 0 & 0 \\
\hline
\end{tabular}


valori che conseguono da $\lambda_{2} J_{1}\left(\omega_{2}\right)-\mu_{2} Y_{1}\left(\omega_{0}\right)$ nelle varie sezioni di $C D$, per il fattore

$$
\frac{A_{2}^{\prime}}{A_{1}^{\prime}} \frac{\alpha_{1}}{\alpha_{2}} \frac{1}{\Delta_{2}}=\frac{\alpha_{1}}{\alpha_{2}} \frac{\beta_{2}}{\beta_{1}} \frac{1}{\Delta_{1}} \frac{\lambda_{1} J_{1}\left(n \beta_{1}\right)-\mu_{1} Y_{1}\left(n \beta_{1}\right)}{\lambda_{2} J_{1}\left(n \beta_{2}\right)-\mu_{2} Y_{1}\left(n \beta_{2}\right)}=k^{\prime}
$$

Infine, la continuità fra i tratti CD e DE si ottiene moltiplicando i valori di $J_{1}\left(\omega_{2}{ }^{\prime}\right)$ in $\mathrm{DE}$ per il fattore

$$
h^{\prime} \cdot \Delta_{2}
$$

dove $k^{\prime}$ è dato dalla [19].

Gli spostamenti orizzontali relativi per le oscillazioni libere con uno, due, tre nodi, calcolati sulla base delle avvertenze che precedono, sono riportati nella tabella III e, in grafico, nelle figg. da 3 a 7 .

3. - Riassumiamo nel seguente prospetto, alcune caratteristiche idrodinamiche delle oscillazioni libere del lago di Scanno, ottenute con il nuovo metodo:

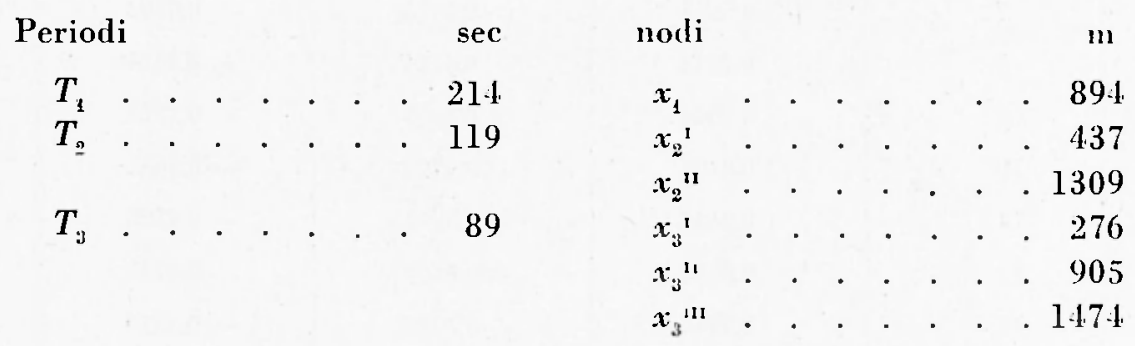

Questi valori sono in ottimo accordo con quelli ottenuti nel lavoro citato.

Sottoporremo i risultati del calcolo al vaglio delle osservazioni dirette.

Roma - Istituto Nazionale di Geofisica - Gennaio 1952.

\section{RIASSUNTO}

L'andamento della curva normale relativa al lago di Scanno, ha suggerito l'opportunità dell'applicazione di un metodo, proposto da Clurystal, per la determinazione delle fondamentali caratteristiche idrodinamiche del lago stesso.

Gli sviluppi analitici ci hanno consentito di pervenire a formule atte alla determinazione dei periodi, dei nodi, degli spostamenti ver- 
ticali e orizzontali, relative alle oscillazioni libere uninodali, binodali e trinodali del lago.

É questa la prima integrale applicazione del metodo proposto da Chrystal, applicazione che ci ha consentito, fra l'altro, di individuare e chiarire un errore, in cui erano incorsi nel 1910 i giapponesi $\mathrm{Na}$ kamura e Honda.

I risultati ottenuti sono in ottimo accordo con quelli tratti in un precedente lavoro.

Ci riserviamo di confrontare $i$ risultati dei calcoli con una serie di registrazioni, che ci proponiamo di ottenere con limnografi, in fase di allestimento.

\section{SUMMARY}

The study of the normal curve shape for the Scamo Lake, has suggested the opportunity of the applicution of the method suggested by Chrystal for the determination of the fundamental hydrodynamical characteristics of the above mentioned Lake.

Analytical developments enabled the A. to obtain formulae which are particularly fit to determine periods, nodes as well as vertical and horizontal displacements in connection to uninodal, binodal and trinodal free oscillations of the Lake.

This study represents the first integral application of the method proposed by Chrystal, and this application enabled the A. to trace and explain at the same time a fault committed in 1910 by the Japaneses Nakamura and Honda.

Results agree perfectly with those obtained in a previous work.

The $A$. will in due time compare the computation results with those he will obtain from a series of records from some limnographic recording apparatus which are presently under manufacturing.

\section{BIBLIOGRAFI $A$}

(1) DI Filirro D.: Le sesse del lago di Scanno. Ann. di Geof., II (19.19).

(2) Calor P.: Le sesse del lago di Garda. Parte II. Ann. di Geof, I (1948).

(3) Nakamula S. e Honda K.: Seiches in Some Lakes of Japan. The Journal of the Coll. of Science, Imp. Univ. Tokyo, XXVIII (1910). 